\title{
Chest pain symptom scoring can improve the quality of referrals to Rapid Access Chest Pain Clinic
}

\author{
Krishnaraj Sinhji Rathod, Helen Ward, Fahad Farooqi
}

Barking Havering Redbridge NHS Trust

\begin{abstract}
Typical stable angina is a clinical diagnosis based on history. The challenge for GPs in primary care is to identify those patients who are presenting with either possible or typical angina symptoms and refer onwards for specialist assessment in the local Rapid Access Chest Pain Clinic (RACPC).

Our initial information gathering study suggested that referring GPs may be cautiously overdiagnosing angina in primary care, potentially resulting in avoidable or unnecessary referrals to RACPC. We sought a practical and cost effective solution to reducing avoidable referrals by assisting GPs with chest pain discrimination.

We tested a change of referral form incorporating chest pain symptom scoring to see whether GP referral quality could be improved and then assessed its impact post implementation. GPs that used the chest pain symptom scoring questionnaire were more than twice as likely to correctly discriminate non-cardiac chest pain. Our post implementation study of the new referral form showed that the proportion of referrals to RACPC with diagnosis of non-cardiac chest pain reduced by almost $19 \%$, and there was a statistically significant $30 \%$ fall in the total number of referrals to RACPC. This was likely to be driven by the deterrent effect of the novel referral form on avoidable referrals. Fewer avoidable referrals results in shorter wait times for specialist review, reduces the risk of waiting time breach, and improves RACPC efficiency.
\end{abstract}

In summary, chest pain symptom scoring resulted in improved GP discrimination of chest pain, improved referral quality, fewer overall referrals to RACPC and shorter patient wait times. These benefits were achieved without using additional financial resources and without the time or capital expense of training GPs. These findings could assist GPs and Clinical Commisioning Groups to achieve cost savings by reducing avoidable secondary care referrals.

\section{Problem}

Chest pain is a common presenting symptom in patients attending general practice, but only a minority of patients will have a diagnosis of stable angina. Patients presenting with atypical chest pain symptoms and no cardiovascular risk factors are very unlikely to have a diagnosis of stable angina and therefore should not be referred to RACPC. These low risk patients are instead best managed in primary care where physicians could reassure them about their heart and arrange alternative investigation, if appropriate.

The RACPC at the King George Hospital (KGH) receives a high volume of referrals from primary care, reviewing up to 1400 patients per year. KGH is a busy District General Hospital located in llford, Essex on the outskirts of North East London. The unusually high service demand could relate to the above average prevalence of cardiovascular risk factors in the local population. However, in recent years cardiology specialists reviewing referrals in RACPC have fed back the perception that a significant proportion of referrals from GPs are "avoidable" or "unnecessary." The KGH RACPC has increasingly struggled to balance service capacity with demand and consequently come perilously close to breaching the 14 day wait limit for referrals.
If avoidable or unnecessary referrals could be deterred, then there is a reasonable expectation that the total number of referrals to RACPC would reduce and that the referral quality would improve resulting in a more efficient use of the RACPC service and shorter patient wait times. Avoidable or unnecessary referrals sent to RACPC has undesirable consequences, and may result in:

- Delays to appropriate patients being seen

- Unnecessary costs for Clinical Commissioning Groups

- Inefficient use of specialist secondary care expertise and resources.

\section{Background}

RACPCs are established in almost all acute trusts in England and Wales. They provide expeditious specialist review, investigation, and treatment of patients with suspected recent onset stable angina and thereby play an essential part in the National Service Framework for coronary artery disease. In the UK, the earliest mention of chest pain clinics was in 1976 (Duncan, Fulton, Morrison et al 1976). In contrast to the chest pain clinics (CPCs), the RACPCs focus on ambulatory chest pain patients. 
Typical stable angina is a diagnosis made principally on history, so taking a detailed history remains the cornerstone of diagnosis. Certain chest pain symptom characteristics have been shown to make a diagnosis of angina unlikely, eg angina is not usually sharp or stabbing in nature, is not usually influenced by respiration, and is not usually fleeting or lasting for hours.

We sought a practical solution to reducing avoidable or unnecessary referrals to RACPC by assisting GPs with chest pain discrimination before submitting referral to RACPC. Our plan was firstly to assess the existing RACPC referral quality, then test a change of referral form (incorporating chest pain symptom scoring questionnaire) to see if GP referral quality could be improved and finally, assess the impact of changes post implementation. This project involved undertaking three studies over the course of 12 months:

Study 1: Preliminary study to assess the quality of RACPC referrals at $\mathrm{KGH}$

Study 2: A pilot study testing the effectiveness of chest pain symptom scoring in improving GP discrimination of chest pain

Study 3: Review of RACPC referrals post implementation of new referral form.

\section{Baseline measurement}

Study 1: Preliminary study to assess the quality of RACPC referrals at $\mathrm{KGH}$

Aim: An initial "information gathering" single centre study to assess the quality of referrals to RACPC by comparing GP clinical assessment of stable angina with specialist cardiology assessment.

Method: All unselected consecutive GP referrals to KGH RACPC seen over the six week period 1st April 2013 to 15th May 2013 were included in the study. Any patients who were referred to RACPC but did not attend their specialist review appointment were excluded from the analysis. The specific data collected on each patient was "GP suspected diagnosis" and "specialist diagnosis." Data was sourced from the clinical details recorded on the RACPC referral form completed by the GP, and the reported diagnosis on correspondence from specialist review and "patient wait time" for specialist review in days (calculated as the number of whole days between receipt of RACPC referral and patient specialist review in RACPC).

Each referred patient was categorised into one of two discrete and mutually exclusive diagnoses, i.e. either typical angina/possible angina or non-cardiac chest pain as determined by referring GP and then specialist opinion (please see figure 1 for study diagnosis definitions). The proportion of patients in each category was calculated as a percentage of the total referrals seen. The number of patients with a GP diagnosis of "non-cardiac chest pain" was compared with the (gold standard) specialist diagnosis to arrive at a correlation statistic defined as a GP:specialist chest pain correlation ratio. A theoretical correlation ratio of 1:1 for non-cardiac chest pain, for example, would indicate that the GP determined diagnosis of non-cardiac chest pain is clinically equivalent to specialist assessment. Chi Square testing of the correlation statistic was performed (the null hypothesis stating that there is no difference between GP and specialist assessment of chest pain symptoms). The proportion of "inappropriate" referrals was also determined. This was defined as the percentage of unnecessary or avoidable referrals with discernible atypical/non-cardiac chest pain and no cardiovascular risk factors; this patient group is discharged from specialist care with reassurance and no further cardiac investigation is planned.

Results: All 167 unselected consecutive patients seen in KGH RACPC over the six week period were included in the analysis (see figure 2). Assuming that the specialist assessment of chest pain is the gold standard, only $45 \%$ of patients referred had typical angina or possible angina. There was substantial overdiagnosis of angina/atypical angina by GPs: $95 \%$ vs. $45 \%$. A correlation statistic of $1: 11$ was found indicating that specialists were eleven times more likely to diagnose non-cardiac chest pain in the patients referred to RACPC than a GP. Chi Square analysis confirmed that chest pain discrimination by GP differed statistically significantly from specialist $(p<0.05)$, i.e. there was no correlation between specialist and GP diagnosis of non-cardiac chest pain in the cohort of patients referred to King George Hospital RACPC. In addition, more than half $(54.2 \%)$ of all referrals to RACPC were deemed by specialist review to have clearly discernible non-cardiac chest pain (i.e. atypical chest pain symptoms with no cardiovascular risk factors). The mean wait time for specialist review was 11 days.

Conclusion: This information gathering study identified a marked mismatch between GP and specialist assessment of chest pain with a statistically significant eleven fold variance in diagnosis of noncardiac chest pain. Over half (55\%) of all RACPC referrals were deemed by specialist review to have clearly discernible non-cardiac chest pain and could have been "avoided" if GP discrimination of chest pain was as good as specialists. The mean wait time for specialist review was 11 days which is close to breaching the wait time limit of 14 days.

See supplementary file: ds4017.docx - "Figures"

\section{Design}

Having completed study 1 and reflected on the findings, we predicted that if referring GPs had assistance with chest pain discrimination then the proportion referred to RACPC with clearly discernible non-cardiac chest pain could be reduced. If significant numbers of "avoidable" referrals were deterred then overall patient wait times for specialist review could also reduce.

We sought a practical solution to reducing avoidable or unnecessary referrals to RACPC by assisting GPs with chest pain discrimination before submitting their referral to RACPC.

Proposed solution

The conventional referral form at King George Hospital RACPC 
(see figure 3, old RACPC referral form), like many UK hospitals, requests free text description of chest pain symptoms, relevant history and risk factor documentation. This referral form, while functionally sound, had a potential weakness in that it does not assist the referring GP to assess the likelihood of angina before making a referral.

We carried out an extensive internet search of online accessible UK RACPC referral forms and discovered an unconventional referral form in current use at University College Hospital, London (UCLH) that incorporates a unique chest pain symptom scoring questionnaire to guide likelihood of angina. The chest pain symptom scoring questionnaire was created by consultant cardiologist Dr Justin Zaman during his PhD research at UCLH and has been validated both as a diagnostic and prognostic tool for the assessment of chest pain. The user-friendly questionnaire can assist GPs in assessing the likelihood of stable angina and potentially guide more appropriate referrals to RACPC.

We proposed that a referral form incorporating chest pain scoring may improve the quality of GP referrals. In order to test this hypothesis, consent was obtained to design and test a new referral form for King George Hospital which was adapted and modified slightly from the existing UCLH version created by Dr Zaman. Informal feedback from three local GPs with special interest in cardiology was sought on the design of the new referral form and came back universally positive for user friendliness and ease of prompt completion.

The referring GP completes the chest pain symptom questionnaire after taking a detailed history from the patient. The total chest pain symptom score is then calculated (points are credited for symptoms consistent with stable angina and points deducted for symptom characteristics which suggest a non-anginal or alternate cause for chest pain).

A low total chest pain symptom score (i.e. $\leq 1$ ) indicates that stable angina is very unlikely and GPs are discouraged from referring to RACPC and advised to seek an alternative cause for chest pain. An intermediate total score of 2 equates to possible atypical angina and requires the presence of at least one conventional cardiovascular risk factor to meet the threshold for referral for assessment RACPC. A total score of 3 or more indicates typical angina symptoms and automatically fufils referral criteria for RACPC. The chest pain symptom score offers GPs an objective and quantitative diagnostic tool to guide the likelihood of angina and appropriateness of referral to RACPC.

GPs are therefore able to judge the likelihood of angina and determine the appropriateness of referral by calculating a total chest pain symptom score during patient consultation. In the next two studies we tested the effectiveness of chest pain symptom scoring in improving GP discrimination of chest pain and then tested the impact on quality of referrals to RACPC.

Study 2. A pilot study testing the effectiveness of chest pain symptom scoring in improving GP discrimination of chest pain. We tested the hypothesis that chest pain symptom scoring could improve RACPC referral quality by assisting GPs to better discriminate angina/possible atypical angina from non-cardiac chest pain and thereby guide more appropriate referrals. This study involved trialling a newly developed form incorporating chest pain scoring questionnaire (See figure 4: novel referral form).

The quality of referrals received on the novel form would be compared with the existing referral form (acting as control). Improvement in referral quality for the purposes of this study was defined as a proportionate reduction in "avoidable" referrals with discernible non-cardiac chest pain. The gold standard diagnostic assessment was again taken to be the cardiology specialist opinion of either typical/possible angina or non-cardiac chest pain.

Method: A head to head comparison of the quality of referrals received on the existing versus the novel referral form was carried out over a six week study period 1st July to 15th Aug 2013. Copies of the novel referral form were randomly distributed to some local referring GPs and each GP was offered a free choice option of either using the existing conventional or novel referral form for prospective referrals to KGH RACPC. GPs were provided with a one month grace period to familiarise themselves with the layout of the new referral form before commencing the study. This study had a similar design to study 1 in that all unselected consecutive GP referrals received and seen at KGH RACPC over a six week period would be included in the analysis.

Specific data was collected on each patient reviewed in RACPC, ie which referral form was used (either novel or conventional), GP assessed diagnosis and specialist assessed diagnosis (data sourced from the clinical details recorded on the RACPC referral form completed by GP and the reported diagnosis on correspondence from specialist review). Direct comparisons were made of the quality of referrals received on the novel referral form versus the conventional referral form using the same correlation ratio calculation as applied in study 1 with Chi Square analysis to test the null hypothesis for statistical significance. The null hypothesis stated that there is no difference between GP and specialist diagnosis of non- cardiac chest pain symptoms.

Results: All 79 unselected consecutive GP referrals to KGH RACPC were included. The mean patient wait time was 9.4 days. Approximately one third (32.5\%) of patient referrals were received on the novel form. GP assessment of chest pain was compared with gold standard cardiologist assessment of chest pain and each patient was categorised into typical/possible angina or non-cardiac chest pain. As in study 1, a correlation ratio was calculated comparing GP diagnosis of non-cardiac chest pain versus cardiologist assessment based on the patient clinical details provided by the GP on the referral form. During the study period, the correlation ratio of GP assessment of non-cardiac chest pain:cardiologist assessment of non-cardiac chest pain was 5:1 using the novel form and 11:1 using the conventional form. Chi Square analysis showed that the 5:1 correlation ratio achieved using the novel form was not statistically significant, while the 11:1 correlation ratio observed with the conventional form was statistically significant. 
Conclusion: There was an unexpected $>50 \%$ percent reduction in the total number of referrals to RACPC seen ( 79 vs 167 when compared with study 1) and the mean wait time was shorter, though an unknown proportion of this variation may be due to seasonal variations in referral numbers.

GPs who used the novel form for referrals were more than twice as likely to correctly determine non-cardiac chest pain as compared with the conventional form. Put another way, there was less "overdiagnosis" of angina/possible angina by GPs who used the novel form ( $88 \%$ vs $58 \%)$.

Chi Square analysis showed that the 5:1 correlation ratio achieved using the novel form was not statistically different to specialist assessment (which failed to reach clinical significance due to small sample size). The 11:1 correlation ratio observed with the conventional form was, however, statistically significant and consistent with the previous findings observed in study 1 .

\section{Strategy}

Having demonstrated quality improvements, there was departmental consensus support for Trust-wide implementation of the new form and withdrawal of the conventional form. Once implemented the effectiveness of the new form could be regularly audited as part of the Trusts' continuous improvement strategy.

Study 3. Review of RACPC referrals post implementation of new referral form

A post implementation analysis to assess the impact of the novel referral form on diagnosis of patients referred to RACPC was undertaken. The referrals numbers and proportion of specialist diagnosis of non-cardiac chest pain were compared before and after implementation.

Method: All GP referrals seen in RACPC over three months (1st February to 30th April 2014) after implementation of the novel referral form. The proportion of non-cardiac chest pain diagnoses were compared with historic data collated over the same calendar quarter in the three immediate preceding years (2011 to 2013) before implementation. The data collected were number of referrals, percentage diagnosed by specialist with non-cardiac chest pain, and average patient wait in days. The data collected over the same calendar quarter in the preceding three years were averaged with mean and standard deviations calculated to enable statistical comparison with actual 2014 data.

\section{Results}

There was $18.6 \%$ relative reduction in expected referrals with a diagnosis of non-cardiac chest pain after implementation of the novel form (did not reach statistical significance) as compared with the same quarterly average over the preceding three years.

This study also demonstrated a mean $30 \%$ fall in expected numbers of referrals in 2014 ; this was statistically significant $(p<0.05)$. As there are no robust means for identifying exactly how many GP referrals were actually deterred, we used a surrogate marker (comparing with the expected numbers of referrals for that same seasonal quarter based on an average over the same quarterly period in the three preceding years). We assume that deterred referrals have low chest pain symptom scores and so are likely to have non-cardiac chest pain diagnosis.

Conclusion: Using a referral form with chest pain scoring questionnaire, there was a trend towards fewer non-cardiac chest pain referrals (approximately 19\%) which suggests an improvement in referral quality. There was also a shorter patient wait time of 9.8 days which didn't quite reach statistical significance (likely due to small sample size). Relative increase in referral of patients with clinical diagnosis of typical angina/possible angina was observed. The substantial $30 \%$ reduction in expected number of referrals was presumed to be driven by a deterrent effect in referral of patients with low chest pain symptom scores.

\section{Lessons and limitations}

1. This was not a randomised controlled study so our findings could occur by chance. Data collection is relatively short duration and in study 1 and 2 this does not take account of the effect of seasonal variations on referral volume

2. It was single centre so findings may not be comparable with other hospitals

3. There was no objective "hard" endpoint: the cardiologists subjective assessment of chest pain is assumed to be the gold standard for chest pain diagnosis of typical/possible angina vs non-cardiac chest pain

4. The new form may require leading questions to be answered in order to determine the likelihood of angina

\section{Conclusion}

In conclusion, our initial study suggested that many GPs may be cautiously "overdiagnosing" angina in primary care, potentially resulting in avoidable or unnecessary referrals to RACPC for specialist assessment. Our second study shows that GPs who use chest pain symptom scoring (incorporated into the RACPC referral form) are more than twice as likely to correctly discriminate noncardiac chest pain.

Our analysis post full implementation of the new referral form, showed that improvement in GP discrimination translated into actual improvement in referral quality. The proportion of referrals to RACPC with specialist diagnosis of "non cardiac chest pain" reduced by almost $19 \%$, and there was a statistically significant $30 \%$ fall in the total number of referrals to RACPC (likely to be driven by the deterrent effect of the novel referral form on avoidable referrals). There was also a trend towards shorter patient wait times for specialist review.

Importantly, the trend towards improvement in GP referral quality was achieved using existing financial resources and without the time or capital expense of training GPs. Fewer "avoidable" referrals 


\section{BMJ Quality Improvement Reports}

results in shorter wait times for specialist review, less risk of breach and ultimately, could assist GPs and Clinical Commissioning Groups to achieve cost savings targets by reducing secondary care referrals.

Our findings will be submitted to the Trust quality, innovation, productivity and prevention (QIPP) programme as we consider that it demonstrates quality improvement and innovation.

\section{References}

1. Fischbacher CM, Bhopal R, Unwin N, White M, Alberti K. The performance of the Rose angina questionnaire in South Asian and European origin populations: a comparative study in Newcastle, UK. Int J Epidemiol 2001 October 1, 2001;30(5):1009-16.

2. Barakat K, Wells Z, Ramdhany S, Mills PG, Timmis AD. Bangladeshi patients present with non-classic features of acute myocardial infarction and are treated less aggressively in east London, UK. Heart 2003 Mar;89(3):276-9.

3. Chaturvedi N, Rai H, Ben-Shlomo Y. Lay diagnosis and health-care-seeking behaviour for chest pain in south Asians and Europeans. Lancet. 1997 Nov 29;350(9091):1578-83.

4. Raczynski JM, Taylor H, Cutter G, Hardin M, Rappaport N, Oberman A. Diagnoses, symptoms, and attribution of symptoms among black and white inpatients admitted for coronary heart disease. Am J Public Health 1994 Jun;84(6):951-6.

5. Meshack AF, Goff DC, Chan W, Ramsey D, Linares A, Reyna R, Pandey D. Comparison of reported symptoms of acute myocardial infarction in Mexican Americans versus non-Hispanic whites (the Corpus Christi Heart Project). Am J Cardiol 1998 Dec 1;82(11):1329-32.

6. Diamond GA. A clinically relevant classification of chest discomfort. J Am Coll Cardiol 1983;1(2 Pt 1):574-5.

7. Wild $S$, McKeigue P. Cross sectional analysis of mortality by country of birth in England and Wales, 1970-92. BMJ $1997 ; 314(7082): 705$.

8. Gray L, Harding S, Reid A. Evidence of divergence with duration of residence in circulatory disease mortality in migrants to Australia. Eur J Public Health 2007 Dec;17(6):550-4.

\section{Declaration of interests}

No conflict of interest to declare.

\section{Acknowledgements}

Dr. Justin Zaman (Consultant Cardiologist and Honorary Senior Lecturer at James Paget University Hospital and University of East Anglia) for designing the chest pain symptom score questionnaire.

Dr Elsya Speechly-Dick (Consultant Cardiologist at UCLH) for consenting to the use of a modified version of UCLH RACPC referral form.

Dr. Honer Kadr (Consultant Cardiologist at BHR Hospitals NHS Trust) and GPs with Specialist Interest in Cardiology: Shabana Ali, Dr Jagen John and Dr Hari Singh for all their constructive feedback and assistance with regional implementation.

Dr. Arif Ahmed (Cardiology Trust Doctor at BHR Hospitals NHS Trust), Mr. Stuart Wright (Cardiac Nurse Specialist) and Mr. Fouad Magho (Cardiac Nurse Specialist) for assistance in data collection and processing.

Dr Helen Ward (Acute Medicine Specialist Registrar) processing data and presenting findings at departmental meeting. 